\title{
Time-dependent rescalings and Lyapunov functionals for some kinetic and fluid models
}

\author{
Jean Dolbeault \\ Ceremade, U.M.R. C.N.R.S. no. 7534, \\ Université Paris IX-Dauphine \\ Place du Maréchal de Lattre de Tassigny \\ 75775 Paris Cédex 16, France
}

April 25, 1999

\begin{abstract}
We apply the method of time-dependent rescalings which has been developed by G. Rein and the author to a model kinetic equation, to the Euler equations for a perfect polytropic gas and to a model with friction and heat transfer. We build Lyapunov functionals which are in the case of the fluid models improved versions of the estimates which have been found by J.-Y. Chemin and D. Serre (see [2], [14]).
\end{abstract}

\section{Introduction}

In this paper, we present on some new examples a method which has been developed by the author and G. Rein (see [7]) to build Lyapunov functionals and to study dispersion effects in kinetic theory and related models. The main idea is to make a time-dependent rescaling of the system which preserves the structure of the equation but adds a confining harmonic force which transforms asymptotically self-similar solutions of the initial problem into asymptotically stationary solutions of the rescaled problem, even if selfsimilar solutions are not well defined, for at least the corresponding initial data. The preservation of the $L^{1}$-norm by this rescaling introduces a friction 
term which decreases the energy of the rescaled system and is therefore a Lyapunov functional of the original system. This Lyapunov functional may be used directly to measure the dispersion effects, or by the means of an interpolation between a moment and an $L^{p}$-norm (with $p>1$ ), or even the entropy (see [6] in the case of the Boltzmann equation and other models with a collision term).

Actually, one can reinterpret the Lyapunov functional (or at least the term which comes out from the rescaled kinetic energy) as a measure of the dispersion around an average velocity $\frac{\dot{R}}{R} x$ which decays to 0 for any fixed $x \in \mathbb{R}^{d}$ as $t \rightarrow+\infty$, and a direct computation allows us to find the expression of $R(t)$ and the corresponding decay. The fact that the average velocity decays to 0 might be surprising at first sight but it essentially means that the velocity of the particles remaining in a fixed bounded region for large times is small.

The time dependent rescaling considered in [7] and in this paper has the interesting property that it does not introduce any singularity at $t=0$ : the initial data for the rescaled problem can be choosen to be the same as for the unscaled system. The rescaled equation therefore connects the initial data to a stationary solution - when the solution converges - which is the asymptotic dispersion profile or, in the language of parabolic equations, the "intermediate asymptotics" (see [3] for the use of entropy methods in the context of porous media and fast diffusion equations).

The purpose of this paper is to present a general method - which seems very efficient in the context of kinetic equations and fluid dynamics - for getting estimates, more than to give new results, which were already known (up to minor improvements), especially in the context of Euler or Navier Stokes equations (see for instance the papers [2] and [14] by J.-Y. Chemin and D. Serre). The fact that similar methods apply in kinetic theory, fluid dynamics and quantum physics is now well known (see [9], [10], [11], [12], [13] for instance). Since we are only looking for a priori estimates, the computations will be done in the context of classical solutions, which are supposed to exist globally in time, and we will not give further justifications to the integrations by parts. The method may be applied to other equations, for instance to nonlinear Schrödinger equations or even parabolic equations, but we will not insist here on these aspects and refer to [7] or [3] for further comments on this aspect of the question. 


\section{Nonlinear Vlasov equation}

Consider the nonlinear Vlasov equation

$$
\begin{gathered}
\partial_{t} f+v \cdot \partial_{x} f-\partial_{x}\left(\rho^{\gamma-1}\right) \cdot \partial_{v} f=0, \\
\rho(t, x)=\int_{\mathbb{R}^{d}} f(t, x, v) d v
\end{gathered}
$$

$t>0, \quad(x, v) \in \mathbb{R}^{2 d}$, which has been studied for instance by I. Gasser, P. Markowich and B. Perthame in [8]. This nonlocal equation provides a very simple example to study the dispersion effects in kinetic theory. As in [7], we may consider the change of variables

$$
d t=A^{2}(t) d \tau, x=R(t) \xi, \eta=\frac{A^{2}(t)}{R(t)}\left(v-\frac{\dot{R}(t)}{R(t)} x\right)
$$

If $F$ and $\nu$ are respectively the rescaled distribution function and the spatial density in the rescaled variables:

$$
f(t, x, v)=G(t) F(\tau, \xi, \eta), \rho(t, x)=\frac{G R^{d}}{A^{2 d}} \nu(\tau, \xi),
$$

then they satisfy the equation

$$
\begin{aligned}
\partial_{\tau} F+\eta \cdot \partial_{\xi} F & +2 A^{2}\left(\frac{\dot{A}}{A}-\frac{\dot{R}}{R}\right) \eta \cdot \partial_{\eta} F \\
- & \ddot{R} \frac{A^{4}}{R} \xi \cdot \partial_{\eta} F-\frac{A^{4}}{R^{2}}\left(\frac{G R^{d}}{A^{2 d}}\right)^{\gamma-1} \partial_{\xi}\left(\nu^{\gamma-1}\right) \cdot \partial_{\eta} F+A^{2} \frac{\dot{G}}{G} F=0 .
\end{aligned}
$$

The invariance of the $L^{1}$-norm by the change of variables - or equivalently the fact that the terms

$$
A^{2} \frac{\dot{G}}{G} F+2 A^{2}\left(\frac{\dot{A}}{A}-\frac{\dot{R}}{R}\right) \eta \cdot \partial_{\eta} F
$$

can be factorized into a term of the form $\partial_{\eta}(\eta F)$ - is given by the condition

$$
G=\left(\frac{A}{R}\right)^{2 d}
$$


If the coefficients of the harmonic force term and of the nonlinear term are constant in time (which in other words means that one chooses the asymptotic time dependent scale in $x$ which preserves the structure of the equation and such that stationary states may exist), then

$$
\frac{A^{4} \ddot{R}}{R}=1 \quad \text { and } \quad \frac{A^{4}}{R^{2}}\left(\frac{G R^{d}}{A^{2 d}}\right)^{\gamma-1}=1
$$

(it is not restrictive to choose the constants to be equal to 1 ). This can be solved into

$$
\begin{gathered}
\ddot{R}=R^{-[(\gamma-1) d+1]}, \\
A=R^{\frac{1}{4}[(\gamma-1) d+2]}, \frac{d \tau}{d t}=R^{-\frac{1}{2}(\gamma-1) d-1}, G=R^{\frac{d}{2}[(\gamma-1) d-2]},
\end{gathered}
$$

and the equation for $F$ is now

$$
\partial_{\tau} F+\eta \cdot \partial_{\xi} F-\xi \cdot \partial_{\eta} F-\partial_{\xi}\left(\nu^{\gamma-1}\right) \cdot \partial_{\eta} F=\frac{1}{2}[(\gamma-1) d-2] R^{\frac{(\gamma-1) d}{2}} \dot{R} \partial_{\eta}(\eta F) .
$$

Note that we may choose $R(0)=1, \dot{R}(0)=0$ and $\tau(0)=0$ :

$$
F(0, \xi, \eta)=f(0, \xi, \eta)
$$

for any $(\xi, \eta) \in \mathbb{R}^{2 d}$. If $\gamma>1$, the solution of $\ddot{R}=R^{-[(\gamma-1) d+1]}$ satisfies $R(t) \sim t$ as $t \rightarrow+\infty$.

An easy computation shows that the energy $L(t)$ given by

$$
\begin{aligned}
L(t)=\frac{1}{2} \iint_{\mathbb{R}^{d} \times \mathbb{R}^{d}}\left(|\eta|^{2}+|\xi|^{2}\right) F(\tau(t), \xi, \eta) d \eta d \xi & \\
+ & \frac{1}{\gamma} \int_{\mathbb{R}^{d}} \nu^{\gamma}(\tau(t), \xi) d \xi \\
= & R^{(\gamma-1) d}(t)\left[\frac{1}{2} \iint_{\mathbb{R}^{d} \times \mathbb{R}^{d}}\left|v-\frac{\dot{R}}{R} x\right|^{2} f(t, x, v) d v d x+\frac{1}{\gamma} \int_{\mathbb{R}^{d}} \rho^{\gamma}(t, x) d x\right] \\
& +\frac{1}{2 R^{2}(t)} \int_{\mathbb{R}^{d}}|x|^{2} \rho(t, x) d x
\end{aligned}
$$

satisfies

$$
\frac{d L}{d t}(t)=[(\gamma-1) d-2] \frac{\dot{R}}{R} \iint_{\mathbb{R}^{d} \times \mathbb{R}^{d}} \frac{|\eta|^{2}}{2} F(\tau(t), \xi, \eta) d \eta d \xi
$$

which provides the following decay estimates 
Theorem 2.1 Assume that $\gamma \in] 1,1+\frac{2}{d}[$. Consider a nonnegative solution $f \in C^{0}\left(\mathbb{R}^{+}, L^{1}\left(\mathbb{R}^{d} \times \mathbb{R}^{d}\right)\right.$ corresponding to an inital datum $f_{0}$ such that $(x, v) \mapsto f(x, v)\left(|x|^{2}+|v|^{2}\right)$ and $x \mapsto \int_{\mathbb{R}^{d}} f_{0}(x, v) d v$ belong to $L^{1}\left(\mathbb{R}^{2 d}\right)$ and $L^{1}\left(\mathbb{R}^{d}\right)$ respectively. Then

$$
\begin{aligned}
t \mapsto R^{(\gamma-1) d}(t)\left[\frac{1}{2} \iint_{\mathbb{R}^{d} \times \mathbb{R}^{d}}\left|v-\frac{\dot{R}}{R} x\right|^{2}\right. & \left.f(t, x, v) d v d x+\frac{1}{\gamma} \int_{\mathbb{R}^{d}} \rho^{\gamma}(t, x) d x\right] \\
& +\frac{1}{2 R^{2}(t)} \int_{\mathbb{R}^{d}}|x|^{2} \rho(t, x) d x
\end{aligned}
$$

with $\ddot{R}=R^{-[(\gamma-1) d+1]}, R(0)=1, \dot{R}(0)=0$, is decreasing.

Remark 2.2 Given any $L^{\infty}\left(\mathbb{R}^{+}, L^{p}\left(\mathbb{R}^{2 d}\right)\right)$ bound on $f$, it is easy to obtain other decay estimates by the interpolation of the $L^{q}\left(\mathbb{R}^{d}\right)$ norm of $\rho(t,$. between $\|f(t, . . .)\|_{L^{p}\left(\mathbb{R}^{2 d}\right)}$ and $\iint_{\mathbb{R}^{d} \times \mathbb{R}^{d}}\left|v-\frac{\dot{R}}{R} x\right|^{2} f(t, x, v) d v d x$ : there exists a constant $C>0$ such that

$$
\|\rho(t, .)\|_{L^{q}\left(\mathbb{R}^{d}\right)} \leq C\left(\|f(t, . . .)\|_{L^{p}\left(\mathbb{R}^{2 d}\right)}\right)^{1-\theta}\left(\iint_{\mathbb{R}^{d} \times \mathbb{R}^{d}}\left|v-\frac{\dot{R}}{R} x\right|^{2} f(t, x, v) d v d x\right)^{\theta}
$$

provided $\frac{1}{q}=d \frac{1-\theta}{p}+(d+2) \theta$ and $1=\frac{1-\theta}{p}+\theta$ (these coefficients are easily recovered with a scaling argument). As a consequence

$$
\|\rho(t, .)\|_{L^{q}\left(\mathbb{R}^{d}\right)}=O\left(t^{-\theta(\gamma-1) d}\right) .
$$

Remark 2.3 Consider the change of variables $(t, x, v) \mapsto\left(t, x, \eta=v-\frac{\dot{R}}{R} x\right)$. $\tilde{F}(t, x, \eta)=f(t, x, v)$ is a solution of

$$
\partial_{t} \tilde{F}+\eta \cdot \partial_{x} \tilde{F}-\frac{\ddot{R}}{R} x \cdot \partial_{\eta} \tilde{F}-\partial_{x}\left(\rho^{\gamma-1}\right) \cdot \partial_{\eta} \tilde{F}+\frac{\dot{R}}{R}\left[\partial_{x}(x \tilde{F})-\partial_{\eta}(\eta \tilde{F})\right]=0
$$

and we may recover Equation (2.1) as follows: the energy associated to the equation for $\tilde{F}$

$$
\begin{aligned}
E(t)= & \iint_{\mathbb{R}^{d} \times \mathbb{R}^{d}} \tilde{F}(t, x, \eta) \frac{|\eta|^{2}}{2} d x d \eta \\
& +\frac{\ddot{R}}{R} \iint_{\mathbb{R}^{d} \times \mathbb{R}^{d}} \tilde{F}(t, x, \eta) \frac{|x|^{2}}{2} d x d \eta+\frac{1}{\gamma} \int_{\mathbb{R}^{d}} \rho^{\gamma}(t, x) d x
\end{aligned}
$$


is such that

$$
\begin{aligned}
\frac{d E}{d t}(t)= & -2 \frac{\dot{R}}{R} \iint_{\mathbb{R}^{d} \times \mathbb{R}^{d}} \tilde{F}(t, x, \eta) \frac{|\eta|^{2}}{2} d x d \eta \\
& +\left(\frac{d}{d t}\left(\frac{\ddot{R}}{R}\right)+2 \frac{\ddot{R}}{R} \frac{\dot{R}}{R}\right) \iint_{\mathbb{R}^{d} \times \mathbb{R}^{d}} \tilde{F}(t, x, \eta) \frac{|x|^{2}}{2} d x d \eta \\
& -\frac{d(\gamma-1)}{\gamma} \frac{\dot{R}}{R} \int_{\mathbb{R}^{d}} \rho^{\gamma}(t, x) d x,
\end{aligned}
$$

and if we write

$$
\frac{d}{d t}\left(R^{q}(t) E(t)\right) \leq 0
$$

we recover Equation (2.1) provided

$$
q=\min (2,(\gamma-1) d) \quad \text { and } \quad \ddot{R}=R^{p} \quad \text { with } \quad p \leq-(q+1) .
$$

\section{Euler Equations for a perfect compressible fluid}

In [7], it has been noticed that estimates for fluid equations can be obtained in a similar way using Remark 2.3 and the case of an Euler equation with a pressure given by $p(t, x)=\rho^{\gamma}(t, x)$ has been analyzed, providing exactly the same kind of results as the nonlinear Vlasov equation, at least for $\|\rho(t, .)\|_{L^{\gamma}\left(\mathbb{R}^{d}\right)}$ bounded.

One has to mention that the close analogy of the dispersion estimates in kinetic theory and in fluid dynamics has been noticed for instance in [13]. This analogy is still true if one interprets the Lyapunov functional as the energy after a time-dependent rescaling, and that is what is investigated here.

We will focus our attention on more realistic models than the nonlinear Vlasov equation and begin with the Euler equations for a perfect polytropic gas

$$
\begin{gathered}
\partial_{t} \rho+\operatorname{div}_{x}(\rho u)=0 \\
\partial_{t}(\rho u)+\operatorname{div}_{x}(\rho u \otimes u)+\nabla_{x} p=0 \\
\partial_{t}\left(\frac{1}{2} \rho|u|^{2}+\rho e\right)+\operatorname{div}_{x}\left(\left(\frac{1}{2} \rho|u|^{2}+\rho e+p\right) u\right)=0
\end{gathered}
$$


where the pressure is given by the law

$$
p=(\gamma-1) \rho e .
$$

The system has been studied by D. Serre (see [14]) and one of the main tools was the computation of dispersion estimates. We shall give here a slightly improved version of these dispersion estimates. Note that these estimates are sufficient to prove the global existence of a solution corresponding to a small initial data, even if larger solutions are known to have a finite existence time interval only (see [14], [15]).

Exactly as in Remark 2.3, we may consider a new velocity variable

$$
\eta(t, x)=u(t, x)-\frac{\dot{R}(t)}{R(t)} x .
$$

Equations (3.1) and (3.2) are transformed into

$$
\begin{gathered}
\partial_{t} \rho+\operatorname{div}_{x}\left(\rho\left(\eta+\frac{\dot{R}}{R} x\right)\right)=0 \\
\partial_{t}(\rho \eta)=\frac{\dot{R}}{R} \operatorname{div}_{x}\left(\rho\left(\eta+\frac{\dot{R}}{R} x\right)\right) x+\left(\frac{\dot{R}^{2}}{R^{2}}-\frac{\ddot{R}}{R}\right)(x \rho)-\operatorname{div}_{x}(\rho u \otimes u)-\nabla_{x} p .
\end{gathered}
$$

Let

$$
E(t)=\frac{1}{2} \int_{\mathbb{R}^{d}} \rho(t, x)|\eta(t, x)|^{2} d x+\int_{\mathbb{R}^{d}} \rho(t, x) e(t, x) d x+A(t) \int_{\mathbb{R}^{d}} \rho(t, x)|x|^{2} d x
$$

According to Equation (3.3),

$$
\begin{gathered}
\frac{d}{d t}\left[\frac{1}{2} \int_{\mathbb{R}^{d}} \rho(t, x)|u(t, x)|^{2} d x+\int_{\mathbb{R}^{d}} \rho(t, x) e(t, x) d x\right]=0 \\
\frac{d E}{d t}(t)=\frac{1}{2} \frac{d}{d t}\left(\int_{\mathbb{R}^{d}} \rho(t, x)|\eta(t, x)|^{2} d x-\int_{\mathbb{R}^{d}} \rho(t, x)\left|\eta(t, x)+\frac{\dot{R}}{R} x\right|^{2} d x\right) \\
\quad+\frac{d}{d t}\left(A(t) \int_{\mathbb{R}^{d}} \rho(t, x)|x|^{2} d x\right) \\
=\frac{d}{d t} \int_{\mathbb{R}^{d}}\left(\left(A-\frac{1}{2} \frac{\dot{R}^{2}}{R^{2}}\right)|x|^{2}-\frac{\dot{R}}{R} \eta \cdot x\right) \rho(t, x) d x \\
=\left(\dot{A}-\frac{\dot{R} \ddot{R}}{R^{2}}+\frac{\dot{R}^{3}}{R^{3}}\right) \int_{\mathbb{R}^{d}} \rho(t, x)|x|^{2} d x-\left(\frac{\ddot{R}}{R}-\frac{\dot{R}^{2}}{R^{2}}\right) \int_{\mathbb{R}^{d}} \rho(t, x) \eta \cdot x d x \\
+\left(A-\frac{1}{2} \frac{\dot{R}^{2}}{R^{2}}\right) \int_{\mathbb{R}^{d}}|x|^{2} \partial_{t} \rho(t, x) d x-\frac{\dot{R}}{R} \int_{\mathbb{R}^{d}} x \cdot \partial_{t}(\rho \eta) d x
\end{gathered}
$$


Using Equations (3.5) and (3.6), we get

$$
\begin{aligned}
\frac{d E}{d t}=\left(\dot{A}-\frac{\dot{R}}{R^{2}}\right. & \left.+\frac{\dot{R}^{3}}{R^{3}}\right) \int_{\mathbb{R}^{d}} \rho(t, x)|x|^{2} d x-\left(\frac{\ddot{R}}{R}-\frac{\dot{R}^{2}}{R^{2}}\right) \int_{\mathbb{R}^{d}} \rho(t, x) \eta \cdot x d x \\
& +\left(2 A-\frac{\dot{R}^{2}}{R^{2}}\right) \int_{\mathbb{R}^{d}} x \cdot\left(\eta+\frac{\dot{R}}{R} x\right) \rho(t, x) d x \\
& +2 \frac{\dot{R}^{2}}{R^{2}} \int_{\mathbb{R}^{d}} x \cdot\left(\eta+\frac{\dot{R}}{R} x\right) \rho(t, x) d x \\
& -\frac{\dot{R}}{R} \int_{\mathbb{R}^{d}} x \cdot\left(\frac{\dot{R}^{2}}{R^{2}}-\frac{\dot{R} \ddot{R}^{2}}{R^{2}}\right) \rho(t, x) x d x \\
& -\frac{\dot{R}}{R} \int_{\mathbb{R}^{d}} \rho(t, x)\left|\eta+\frac{\dot{R}}{R} x\right|^{2} d x-d \frac{\dot{R}}{R} \int_{\mathbb{R}^{d}} p(t, x) d x \\
=(2 A- & \left.\frac{\ddot{R}}{R}\right) \int_{\mathbb{R}^{d}} \rho(t, x) x \cdot \eta d x-2 \frac{\dot{R}}{R} \int_{\mathbb{R}^{d}} \rho(t, x) \frac{|\eta|^{2}}{2} d x-d \frac{\dot{R}}{R} \int_{\mathbb{R}^{d}} p(t, x) d x \\
& \left(\dot{A}+2 A \frac{\dot{R}}{R}\right) \int_{\mathbb{R}^{d}} \rho(t, x)|x|^{2} d x .
\end{aligned}
$$

Assuming now that $A=\frac{\ddot{R}}{2 R}$, we are left with

$$
\begin{aligned}
\frac{d E}{d t}=-2 \frac{\dot{R}}{R} \int_{\mathbb{R}^{d}} \rho(t, x) \frac{|\eta|^{2}}{2} d x & -d \frac{\dot{R}}{R} \int_{\mathbb{R}^{d}} p(t, x) d x \\
& +\frac{1}{2}\left(\frac{d}{d t}\left(\frac{\ddot{R}}{R}\right)+2 \frac{\dot{R} \ddot{R}}{R^{2}}\right) \int_{\mathbb{R}^{d}} \rho(t, x)|x|^{2} d x .
\end{aligned}
$$

If we write $L(t)=R^{q}(t) E(t)$ and if we express $p(t, x)$ in terms of $\rho(t, x) e(t, x)$ according to Equation (3.4), then

$$
\begin{aligned}
\frac{d L}{d t}= & (q-2) R^{q-1} \dot{R} \int_{\mathbb{R}^{d}} \rho(t, x) \frac{|\eta|^{2}}{2} d x \\
& +(q-(\gamma-1) d) R^{q-1} \dot{R} \int_{\mathbb{R}^{d}} \rho(t, x) e(t, x) d x \\
& +\frac{1}{2}\left(R^{q} \frac{d}{d t}\left(\frac{\ddot{R}}{R}\right)+(q+2) \dot{R} \ddot{R} R^{q-2}\right) \int_{\mathbb{R}^{d}} \rho(t, x)|x|^{2} d x .
\end{aligned}
$$

The two first coefficients are nonpositive provided

$$
q \leq \min (2,(\gamma-1) d)
$$


If we make the ansatz

$$
\ddot{R}=R^{p}
$$

the coefficient of the last term can be written as

$$
\frac{1}{2}(p+q+1) R^{p+q-2} \dot{R}
$$

and $t \mapsto L(t)$ is decreasing if $p=-(q+1)$. For any $\gamma>1$, we recover the results due to J.-Y. Chemin and D. Serre (see [2], [14]) with constants which are explicit in terms of the initial data, and an additional term $\int_{\mathbb{R}^{d}} \rho(t, x)|x|^{2} d x$ (with a coefficient vanishing as $t \rightarrow+\infty$ ).

Theorem 3.1 If $\gamma>1$, any classical solution of Equations (3.1)-(3.4) is such that

(i) If $1<\gamma \leq 1+\frac{2}{d}$, then

$$
\begin{aligned}
& R^{(\gamma-1) d} \int_{\mathbb{R}^{d}} \rho(t, x)\left(\frac{1}{2}\left|u(t, x)-\frac{\dot{R}}{R} x\right|^{2}+e(t, x)\right) d x+\frac{1}{2 R^{2}} \int_{\mathbb{R}^{d}} \rho(t, x)|x|^{2} d x \\
& =\int_{\mathbb{R}^{d}} \rho(0, x)\left(\frac{1}{2}|u(0, x)|^{2}+e(0, x)\right) d x+\frac{1}{2} \int_{\mathbb{R}^{d}} \rho(0, x)|x|^{2} d x \\
& -\frac{2-(\gamma-1) d}{2} \int_{0}^{t} R^{(\gamma-1) d-1} \dot{R}(s)\left(\int_{\mathbb{R}^{d}} \rho(s, x)\left|u(s, x)-\frac{\dot{R}(s)}{R(s)} x\right|^{2} d x\right) d s .
\end{aligned}
$$

(ii) If $\gamma \geq 1+\frac{2}{d}$, then

$$
\begin{aligned}
& R^{2} \int_{\mathbb{R}^{d}} \rho(t, x)\left(\frac{1}{2}\left|u(t, x)-\frac{\dot{R}}{R} x\right|^{2}+e(t, x)\right) d x+\frac{1}{2 R^{2}} \int_{\mathbb{R}^{d}} \rho(t, x)|x|^{2} d x \\
& =\int_{\mathbb{R}^{d}} \rho(0, x)\left(\frac{1}{2}|u(0, x)|^{2}+e(0, x)\right) d x+\frac{1}{2} \int_{\mathbb{R}^{d}} \rho(0, x)|x|^{2} d x \\
& -\frac{1}{2}((\gamma-1) d-2) \int_{0}^{t} R \dot{R}(s)\left(\int_{\mathbb{R}^{d}} \rho(s, x) e(s, x) d x\right) d s .
\end{aligned}
$$

Here $R$ is the solution of

$$
\ddot{R}=R^{-(q+1)}, \quad R(0)=1, \quad \dot{R}(0)=0
$$

and $(\gamma>1)$

$$
q=\min (2,(\gamma-1) d)
$$


Exactly as in [14], the method also applies to the case of a viscous fluid with heat conduction

$$
\begin{gathered}
\partial_{t} \rho+\operatorname{div}_{x}(\rho u)=0 \\
\partial_{t}(\rho u)+\operatorname{div}_{x}(\rho u \otimes u)+\nabla_{x} p=\operatorname{Div} T \\
\partial_{t}\left(\frac{1}{2} \rho|u|^{2}+\rho e\right)+\operatorname{div}_{x}\left(\left(\frac{1}{2} \rho|u|^{2}+\rho e+p\right) u\right)=\operatorname{div}(T u)+\operatorname{div} q
\end{gathered}
$$

where $T_{i j}$ satisfies Newton's law

$$
T_{i j}=\alpha(\rho)\left(\partial_{i} u_{j}+\partial_{j} u_{i}\right)+\beta(\rho)(\operatorname{div} u) \delta_{i j} .
$$

Assuming now that $A=\frac{\ddot{R}}{2 R}$, we are left with

$$
\begin{aligned}
\frac{d E}{d t}=-2 \frac{\dot{R}}{R} \int_{\mathbb{R}^{d}} \rho(t, x) \frac{|\eta|^{2}}{2} d x & -d \frac{\dot{R}}{R} \int_{\mathbb{R}^{d}} p(t, x) d x \\
& +\frac{1}{2}\left(\frac{d}{d t}\left(\frac{\ddot{R}}{R}\right)+2 \frac{\dot{R} \ddot{R}}{R^{2}}\right) \int_{\mathbb{R}^{d}} \rho(t, x)|x|^{2} d x \\
& -\frac{\dot{R}}{R} \frac{d}{d t} \int_{\mathbb{R}^{d}} g(\rho(t, x)) d x .
\end{aligned}
$$

where $g$ satisfies

$$
g(0)=0, \quad \rho g^{\prime}(\rho)-g(\rho)=2 \alpha(\rho)+\beta(\rho)
$$

$L(t)=R^{q}\left(E(t)+\frac{\dot{R}}{R} \int_{\mathbb{R}^{d}} g(\rho(t, x)) d x\right)$ is then decreasing as soon as

$$
\frac{d}{d t}\left(R^{q-1} \dot{R}\right) \leq 0
$$

If $\ddot{R}=R^{p}$, with $p \leq-(q+1)$, then

$$
\frac{d}{d t}\left(R^{q-1} \dot{R}\right)=(q-1) R^{q-2} \dot{R}^{2}+R^{p+q-1} \sim(q-1) R^{q-2} \dot{R}^{2} \rightarrow-\infty \quad \text { as } t \rightarrow+\infty
$$

provided $q<1$. As a consequence, we may recover the dispersion results given by D. Serre in [14] (for the special case $g(\rho)=C \rho^{\delta}$ with $\delta>1$ ), extend them to the general case and provide an additional momentum $\int_{\mathbb{R}^{d}} \rho(t, x)|x|^{2} d x$.

Theorem 3.2 If $\gamma>1$, any classical solution of Equations (3.7)-(3.10) is such that 
(i) If $1<\gamma<1+\frac{1}{d}$, then

$$
\begin{aligned}
& R^{(\gamma-1) d} \int_{\mathbb{R}^{d}} \rho(t, x)\left(\frac{1}{2}\left|u(t, x)-\frac{\dot{R}}{R} x\right|^{2}+e(t, x)\right) d x \\
& \quad+R^{(\gamma-1) d} \int_{\mathbb{R}^{d}} g(\rho(t, x)) d x+\frac{1}{2 R^{2}} \int_{\mathbb{R}^{d}} \rho(t, x)|x|^{2} d x \\
& =\int_{\mathbb{R}^{d}} \rho(0, x)\left(\frac{1}{2}|u(0, x)|^{2}+e(0, x)\right) d x \\
& +\int_{\mathbb{R}^{d}} g(\rho(0, x)) d x+\frac{1}{2} \int_{\mathbb{R}^{d}} \rho(0, x)|x|^{2} d x \\
& -\frac{2-(\gamma-1) d}{2} \int_{0}^{t} R^{(\gamma-1) d-1} \dot{R}(s)\left(\int_{\mathbb{R}^{d}} \rho(s, x)\left|u(s, x)-\frac{\dot{R}(s)}{R(s)} x\right|^{2} d x\right) d s \\
& -\int_{0}^{t}\left[\frac{1}{R^{2}(s)}-(1-(\gamma-1) d) R^{(\gamma-1) d-2}(s) \dot{R}^{2}(s)\right]\left(\int_{\mathbb{R}^{d}} g(\rho(s, x)) d x\right) d s,
\end{aligned}
$$

where $R$ is the solution of

$$
\begin{aligned}
& \ddot{R}=R^{-[(\gamma-1) d+1]}, \quad R(0)=1, \quad \dot{R}(0)=0 \\
& \left(R(t) \sim t, \frac{\dot{R}(t)}{R(t)} \sim \frac{1}{t} \text { as } t \rightarrow+\infty\right) .
\end{aligned}
$$

(ii) If $\gamma \geq 1+\frac{1}{d}$, then for any $t, t_{0} \in \mathbb{R}$,

$$
\begin{gathered}
t \int_{\mathbb{R}^{d}} \rho(t, x)\left(\frac{1}{2}\left|u(t, x)-\frac{1}{t} x\right|^{2}+e(t, x)\right) d x \\
\quad+t \int_{\mathbb{R}^{d}} g(\rho(t, x)) d x+\frac{1}{2 t^{2}} \int_{\mathbb{R}^{d}} \rho(t, x)|x|^{2} d x \\
=t_{0} \int_{\mathbb{R}^{d}} \rho\left(t_{0}, x\right)\left(\frac{1}{2}\left|u\left(t_{0}, x\right)-\frac{1}{t_{0}} x\right|^{2}+e\left(t_{0}, x\right)\right) d x \\
\quad+t_{0} \int_{\mathbb{R}^{d}} g\left(\rho\left(t_{0}, x\right)\right) d x+\frac{1}{2 t_{0}^{2}} \int_{\mathbb{R}^{d}} \rho\left(t_{0}, x\right)|x|^{2} d x \\
-\int_{t_{0}}^{t}\left(\frac{1}{2} \int_{\mathbb{R}^{d}} \rho(s, x)\left|u(s, x)-\frac{1}{s} x\right|^{2} d x\right. \\
\left.\quad+[(\gamma-1) d-1] \int_{\mathbb{R}^{d}} \rho(s, x) e(s, x) d x\right) d s,
\end{gathered}
$$

Note that in the case $\gamma \geq 1+\frac{1}{d}, R(t)=t$ is exactly the function which is used by D. Serre in [14] (for $g(\rho)=C \rho^{\delta}$ with $\delta>1$ ) and that all the above estimates are equivalent to the ones of D. Serre and J.-Y. Chemin as $t \rightarrow+\infty$. 


\section{Conclusion}

Extracting the right scale and finding the rate of dispersion or even the Lyapunov functional that governs this rate is probably the easy part of the study of the asymptotic dispersion profile or "intermediate asymptotics", and the next (open) question is to understand in which cases the difference of the rescaled solution with a stationary solution of the rescaled equation has a faster, eventually exponential, decay in this framework of a priori estimates. The Lyapunov functional can indeed be used to study the dynamical stability, and in some cases is a relative to the stationary states entropy. This question is not easy, and has been partially answered in the context of systems where the field is coupled to the spatial density by the (coulombic) Poisson equation: the rescaled Vlasov-Poisson system converges to its unique stationary state if $d=1$ (see [1]) but counter-examples to the convergence of the solution to the rescaled Euler-Poisson system to its unique stationary state were found in $[7]$ when $d=3$.

Acknowledgements. The author thanks the program on Charged Particle Kinetics at the Erwin Schroedinger Institute and the TMR "Asymptotic methods in kinetic equations" (Contract ERB FMRX CT97 0157) for partial support.

\section{References}

[1] J. Batt, M. Kunze, G. Rein, On the asymptotic behaviour of a onedimensional, monocharged plasma and a rescaling method, Advances in Differential Equations, to appear.

[2] J.-Y. Chemin, Dynamique des gaz à masse totale finie [Dynamics of gases with finite total mass], Asymptotic Anal. 3 no. 3 (1990) 21-220.

[3] M. del Pino, J. Dolbeault, Long time bahaviour of the solutions to porous medium and fast diffusion equations, in preparation.

[4] J. Dolbeault, Monokinetic charged particle beams: Qualitative behavior of the solutions of the Cauchy problem and $2 d$ time-periodic solutions of the Vlasov-Poisson system, J. Math. Pures Appl. 78 (1999) 121-157. 
[5] J. Dolbeault, Free energy and solutions of the Vlasov-Poisson-FokkerPlanck system: external potential and confinement (large time behavior and steady states) to appear in Journal de Mathématiques Pures et Appliquées

[6] J. Dolbeault, Time-dependent rescalings and dispersion for the Boltzmann equation, Preprint Ceremade no. 9845, to appear in Arch. Rat. Mech. Anal.

[7] J. Dolbeault, G. Rein, Time-dependent rescalings and Lyapunov functionals for the Vlasov-Poisson and Euler-Poisson systems, and for related models of kinetic equations, fluid dynamics and quantum physics, in preparation.

[8] I. Gasser, P. Markowich, B. Perthame, Dispersion and moment lemmas revisited, Preprint.

[9] R. Illner, Existence, uniqueness and asymptotic behavior of WignerPoisson and Vlasov-Poisson systems: a survey, Proceedings of the Fourteenth International Conference on Transport Theory (Beijing, 1995), Transport Theory Statist. Phys. 26 no. 1-2 (1997) 195-207.

[10] R. Illner, H. Lange, P. Zweifel, Global existence, uniqueness and asymptotic behaviour of solutions of the Wigner-Poisson and SchrödingerPoisson systems, Math. Methods Appl. Sci. 17 no. 5 (1994) 349-376.

[11] R. Illner \& G. Rein, The time decay of the solutions of the VlasovPoisson system in the plasma physical case, Math. Methods Appl. Sci. 19 no. 17 (1996) 1409-1413.

[12] B. Perthame, Time decay, propagation of low moments and dispersive effects for kinetic equations, Comm. P.D.E. 21 (1\& 2) (1996) 659-686.

[13] B. Perthame, Décroissance en temps: analogie entre les équations du transport cinétique, de Schrödinger et de la dynamique des gaz, Preprint.

[14] D. Serre, Solutions classiques globales des quations d'Euler pour un fluide parfait compressible [Classical global solutions of the Euler equations for a compressible perfect fluid] Ann. Inst. Fourier (Grenoble) 47 no. 1 (1997) 139-153. 
[15] T.C. Sideris, Formation of singularities in three-dimensional compressible fluids, Comm. Math. Phys. 101 no. 4 (1985) 475-485. 